\title{
Formación, subjetividad y escuela: aproximación fenomenológica al tiempo escolar
}

\author{
Formation, subjectivity and school: \\ a phenomenological approach to school time
}

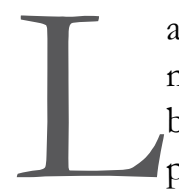

a formación es uno de los temas de mayor importancia no sólo en los debates educativos, sino que constituye el problema fundamental de la escuela en el discurrir del tiempo; en la medida que es el supuesto esencial sobre el cual se movilizan todas las intensiones educativas en el contexto escolar. $\mathrm{Al}$ ser la formación el centro de la actividad educativa en la escuela, implica una mirada no sólo desde las distintas teorías, sino también desde las experiencias de sentido que tiene para quienes hacemos parte de los procesos educativos. El propósito del presente texto es realizar una aproximación en perspectiva fenomenológica al tiempo escolar, a partir de los aportes de Husserl en su texto Lecciones de fenomenología de la conciencia interna del tiempo, editado por Heidegger en 1928.

El desarrollo argumentativo del escrito tiene tres momentos: primero, aproximación a las lecciones de la conciencia interna del tiempo, que busca desarrollar de manera muy breve los conceptos más importantes de Husserl en su esfuerzo por demostrar desde el análisis fenomenológico de la conciencia del tiempo interno como fundamento constitutivo del tiempo objetivo; segundo, el tiempo escolar, aquí se presenta un perspectiva fenomenológica para pensar la formación en el contexto de la escuela, y tercero, formación y subjetividad, en esta parte del texto se desarrollan algunas aproximaciones al tema de la formación y la constitución de subjetividad desde el desarrollo de las tecnologías de la información y las comunicaciones.

\section{Aproximación a las lecciones de la conciencia interna del tiempo}

En ocasiones decimos que el tiempo pasa y, en ocasiones hacemos eco de las palabras que dice el poeta: ;O tempora, o mores! (¡Oh tiempos, oh costumbres!). Surgen preguntas sobre si lo que pasa es el tiempo o son los objetos, lo que hace pensar en la relación que existe entre el tiempo del mundo y la conciencia que tenemos del tiempo y, este es el centro del trabajo de Husserl, "nuestro propósito es un análisis fenomenológico de la conciencia del tiempo" (Husserl, 2002, p. 26), evidenciando con ello la relación entre subjetividad y tiempo. En otras palabras, Husserl busca

Recibido el 3 de julio de 2012 y aprobado el 24 de septiembre de 2012.

1 Víctor Eligio Espinosa Galán es Profesional y Licenciado en Filosofía de la Corporación Universitaria Minuto de Dios, Magister en Desarrollo Educativo y Social de la Universidad Pedagógica Nacional, estudiante del Doctorado Interinstitucional Universidad Pedagógica Nacional.

Polisemia No. 13, 94 - 101. Formación, subjetividad y escuela: aproximación fenomenológica al tiempo escolar. Bogotá, ISSN: 1900-4648. Enero - junio de 2012 
demostrar cómo se constituye el tiempo objetivo en la conciencia del tiempo (Klaus, 2005, p. 10).

El aspecto importante de señalar en el trabajo de Husserl es la vivencia de la temporalidad, donde también tiene origen el tiempo objetivo. En este sentido, vemos que el trabajo de Husserl se encuentra con dos tradiciones filosóficas sobre el tiempo: la primera, se relaciona con las ideas de Aristóteles, para quien el tiempo está estrechamente vinculado al movimiento continuo de las cosas, dado que no es posible pensar el tiempo sin seres en movimiento. Es la conciencia del cambio la que hace que tengamos conciencia del transcurrir del tiempo; por otro lado, se encuentra con las ideas de San Agustín, quien vincula el tiempo al alma, separándolo del movimiento. El tiempo se da en la vivencia de la propia temporalidad del alma:

\begin{abstract}
¿Qué es, pues, el tiempo? Si nadie me lo pregunta, lo sé; pero si quiero explicárselo al que me lo pregunta, no lo sé. Lo que sí digo sin vacilación es que sé que si nada pasase no habría tiempo pasado y si nada sucediese, no habría tiempo futuro; y si nada existiese, no habría tiempo presente. Pero aquellos dos tiempos, pretérito y futuro, ¿cómo pueden ser, si el pretérito ya no es y el futuro todavía no es? Y en cuanto al presente, si fuese siempre presente y no pasase a ser pretérito, ya no sería tiempo, sino eternidad. Si, pues, el presente, para ser tiempo es necesario que pase a ser pretérito, ¿cómo deciros que existe éste, cuya causa o razón de ser está en dejar de ser, de tal modo que no podemos decir con verdad que existe el tiempo sino en cuanto tiende a no ser?” (Agustín, 2001, pp.14-17).
\end{abstract}

En la fenomenología podemos decir que no hay cosas que duran, hay conciencia de las cosas que duran, lo que admite la existencia de un tiempo, que no es el tiempo del mundo, "sino del tiempo interno del curso de la conciencia" (Garrido-Maturano, 2006, p. 3), "hay quien puede determinarse el tiempo objetivo de una vivencia, incluido el de una vivencia que constituye tiempo" (Husserl, 2002, p. 26), es decir, que para nuestro autor el tiempo es tiempo de la duración inmanente de las vivencias en la conciencia.

Es importante tener en cuenta que para Husserl no existe una desconexión definitiva entre el tiempo subjetivo y el tiempo objetivo y, muchos menos, que la fenomenología lo ignore, dado que le interesa a este autor responder a la pregunta por el tiempo, el origen del tiempo objetivo, sólo que orienta su pregunta al origen de la conciencia, en donde cree ver "las fuentes originarías de todas las evidencias relativas al tiempo [...] Nos interesan las vivencias de tiempo $[. .$.$] nos interesa que en$ estas vivencias se mientan datos 'objetivamente temporales" (Husserl, 2002, p. 31).

Siguiendo este breve análisis de la conciencia interna del tiempo, el ejemplo que presenta Husserl es el de la duración de un sonido, como puro dato hilético ${ }^{1}$, no como onda sonora (mundo físico) ni como melodía (mundo percibido): "El sonido comienza y acaba, y a su fin la unidad de toda su duración [...] 'retrocede' a un pasado cada vez más y más lejano”, es decir, que el sonido emerge en un momento determinado y se hunde en el instante siguiente, pero esto no significa que el sonido se hunda en la nada absoluta, el primer sonido continúa a modo de recuerdo, es decir, lo tengo retenido: "y mientras la retención pervive, el sonido posee su temporalidad propia, es el mismo sonido y su duración es la misma" (Husserl, 2002, p. 46).

Impresión, retención y protención constituyen los proactos de la conciencia del tiempo, los cuales hacen que la conciencia pase de un modo continuo de un ahora a otro y posibiliten que en el flujo puedan durar los objetos, en el ejemplo del sonido podríamos ilustrar los protoactos como, protención en tanto sonido esperado por los sonidos que le precedieron, impresión originaria, como ahora viviente y retención como recién sido.

1 En la nota marginal del texto, el autor señala que el dato hilético: "es una denominación adicional de los contenidos primario, sean sensaciones o fantasmas. No se analiza, pues, ni el sonido como unidad causada y causal dentro de la realidad física (onda sonora), ni el sonido como objeto del mundo percibido (la nota de la melodía), sino el sonido como sensación inmanente que forma parte de la percepción auditiva de la nota [cursivas propias] (Husserl,2002, p. 46). 
Cuando Husserl presenta la noción de rememoración, señala que las retenciones se van convirtiendo en intensiones vacías a lo que nos presenta la noción de rememoración o recuerdo secundario y constituye una representación que no está unida al presente impresional:

La rememoración, que se caracteriza, entonces, por volver hacer presente o presentificar en el ahora un objeto que ha sido percibido en el pasado y cuyo contenido intuitivo no está dado como modificación retencional del actual punto fuente (Garrido-Maturano, 2006, p. 11).

En la segunda sección, Husserl desea avanzar en un análisis y presenta el tema problemático del lugar del tiempo, es decir, que en la retención no sólo se retiene lo percibido, sino también se retiene el lugar del tiempo en el que se percibió lo percibido. Es así que el trabajo de Husserl nos va llevando de la desconexión del tiempo objetivo, para mostrar, desde el análisis fenomenológico de la conciencia del tiempo interno, el fundamento constitutivo del tiempo desconectado. Es muy importe la noción de lugar del tiempo, en la medida que existe un flujo que está siempre en retroceso: "al hundirse, el objeto cambia constantemente su lugar en el tiempo, y al hundirse debería más bien preservar su lugar en el tiempo" (Husserl, 2002, p. 84). A renglón seguido, nuestro autor señala que en el continúo retroceso del objeto del recuerdo primario no cambia su lugar en el tiempo, sólo se distancia del ahora actual, que es punto objetivo de tiempo siempre nuevo, "mientras que lo temporal que ha pasado permanece siendo lo que es" (Husserl, 2002, p. 84) le permite afirmar que es el lugar de tiempo: "el que viene a constituir individualidad" (Husserl, 2002, p. 87), en la medida que puede recubrir constantemente un contenido como un contenido fijado en el flujo.

En el esfuerzo de Husserl por fundar el tiempo objetivo en el flujo de la conciencia interna del tiempo, nos presenta en la tercera sección el tema de la conciencia absoluta del tiempo, es decir, que si la conciencia es fuente fenomenológica originaria y constitutiva del tiempo, es necesario que ésta se autoconstituya y autoafirme:
En el flujo absoluto de la conciencia es posible toda duración [...] la duración no es intrínseca a los objetos, si no a los fenómenos en cuanto correlato de una conciencia que fluye y cuyo fluir hace del aparecer del objeto una continuidad de escorzamientos (Garrido-Maturano, 2006, p. 20).

El flujo constituyente de tiempo como conciencia absoluta de tiempo Husserl lo señala como:

Este flujo, este rio, es algo que denominamos así, según lo constituido, pero que no es nada objetivo en el tiempo. Es la subjetividad absoluta, y tiene las propiedades absolutas de lo que en imagen designamos como flujo, rio, como algo que brota ahora en un punto de actualidad, punto que es fuente primigenia [...] para todo esto nos faltan nombres (Husserl, 2002, p. 95).

Finalmente y con la necesidad de cerrar este recorrido — tal vez espinoso- por algunos de los temas de las lecciones, señalo que la conciencia constitutiva del tiempo se convierte en una instancia última de la propia subjetividad, que hace parte de la totalidad de sus actos, con arreglo a una peculiar estructura intencional que tiene la forma del flujo. $Y$ es también esa peculiar organización de la conciencia interna del tiempo como flujo la que garantiza su unidad y su autoaparecer, al hacer de las retenciones no sólo retenciones de sus objetos (intencionalidad transversal del flujo), sino retenciones de retenciones pasadas (intencionalidad longitudinal):

En el flujo de conciencia uno, único, se entrelazan dos intencionalidades inseparablemente unitarias, que se requieren una a la otra como dos caras de una y la misma cosa. Por la primera de ellas se constituye un tiempo inmanente, un tiempo objetivo, auténtico tiempo, en el cual hay duración y hay cambio de lo que dura. En la otra intencionalidad se constituye la ordenación quasi-temporal de las fases del flujo, que tiene siempre y necesariamente un punto - «ahora» fluyente, la fase de actualidad y la serie de fases preactuales y postactuales -las que todavía no son actuales-. Esta temporalidad prefenoménica, preinmanente, se constituye intencionalmente como forma de la conciencia tempoconstituyente y lo hace en la propia conciencia (Husserl, 2002, pp.102-103).

Polisemia No. 13, 94 - 101. Formación, subjetividad y escuela: aproximación fenomenológica al tiempo escolar. Bogotá, ISSN: 1900-4648. Enero - junio de 2012 


\section{El tiempo escolar}

Cuando se trae a la memoria la escuela y las vivencias allí prologadas en el flujo de la propia subjetividad, se entiende que la experiencia humana está íntimamente sujetada al tiempo, a la propia vivencia de la temporalidad en la que se constituye la individualidad, lo singular, la temporalidad absoluta, en donde el sujeto no sólo tiene su experiencia del presente, sino también del pasado y del futuro.

Pero, ¿qué significa el tiempo en la escuela? Recordar el sonido de la campana y la vivencia que tenemos de este sonido en cada uno de los espacios de la escuela, que marcaron multiplicidad de vivencias, pero que en cada tiempo objetivo hacia emerger el tiempos subjetivo, tal vez el único tiempo. No era lo misma la campana para salir a descanso que la campaña para entrar a clase de matemáticas o la campana para ir a la casa. Todas la experiencias del tiempo escolar, como constitución de subjetividad, como un otorgar sentido, se da sólo en la temporalidad, en el horizonte personal, en el soy en primera persona, en ese hacerse sujeto del mundo, en donde la formación sería como un hacerse cargo de la propia temporalidad y la escuela como horizonte común en el que cada uno la vive, no como espacio, sino como lugar desde una perspectiva individual.

Es así que más allá de las teorías sobre el aprendizaje, la formación, el currículo, la disciplina, cada quien constituye su temporalidad en una negociación permanente de sentido. La vivencia del tiempo escolar permite reconocer la distinción entre el tiempo objetivo, en el que no hay una desconexión con el tiempo subjetivo, pero que cede su origen al flujo de la conciencia interna del tiempo.

En este sentido podemos decir, desde lo expuesto en las Lecciones, que el tiempo es relativo a las vivencias que tenemos de él. Constituyéndose así la escuela en el ámbito en el que se realiza la experiencia humana. Cuando se habla de la escuela como lugar de sentido, y como horizonte de sentido, pareciera que está lejos de esto y que por el contrario es un escenario agonístico de sentido, no solo individual sino compartido. Por ello se hace necesario fenomenologizar la escuela, es decir, mirarla como un lugar en que se constituye el sentido, hacerse dueño de la capacidad de construir sentido, más allá de las determinaciones y los esfuerzos totalizantes por constreñir la subjetividad.

Entonces, la pregunta por la formación en la escuela va más allá de cualquier intensión, dirección, orientación o enfoque. La esencia de la formación es experiencia que tiene que estar formándose: ¿qué sentido se ha constituido en el espacio y el tiempo escolar? Pero, ¿cómo entender el cumulo de vivencias y simultaneidades que habitan el espacio escolar?, ¿cómo hacer para ubicar el quehacer de la escuela desde un sentido afirmativo, en el que se construyan las vivencias del ideal de la educación como humanización? En el que, a la vez que hay dación de sentido individual, podamos encontrar en la escuela un lugar de sentido compartido.

Entender éticamente la vida significa darse cuenta de que existir es vivir en un ámbito espacio-temporal, y de que el tiempo es el otro: "el otro presente, pasado y futuro (antepasados, contemporáneos y sucesores) $[\ldots]$ vivir es ser en situación, estar en relación con los otros, con el mundo y en el mundo" (Mélich, 2004. p, 65).

He aquí una de las nociones importantes del tiempo escolar que no es únicamente un tiempo de la vivencia singular, sino que, siendo vivencia singular, está necesariamente unida a la experiencia de estar con otros. Habitar y compartir la escuela como lugar. Por ello señala Mélich que la filosofía fenomenológica de Husserl ha situado la intencionalidad en la base de su reflexión: "toda conciencia es conciencia de algo". En este sentido, es necesario dar un paso de la conciencia individual de Husserl y entender la conciencia del tiempo escolar como conciencia intersubjetiva, porque no es sólo conciencia de mi mismo, sino conciencia de los otros que no son objetos, si no enteramente otros.

\section{Formación y subjetividad}

El tema de la formación es el centro de la actividad educativa en tanto el currículo está 
orientado al desarrollo de las dimensiones de la persona. Es decir, que cuando hablamos de una formación integral estamos colocando en el centro de los procesos de aprendizaje la idea de humanización, no sólo del conocimiento sino de la vida misma.

El concepto de formación, según Flórez,

desarrollado inicialmente en la Ilustración, no es hoy día operacionalizable ni sustituible por habilidades y destrezas particulares ni por objetivos específicos de instrucción. Mas bien los conocimientos, aprendizajes y habilidades son apenas medios para formarse como ser espiritual. La formación es lo que queda, es el fin perdurable; a diferencia de los demás seres de la naturaleza, 'el hombre no es lo que debe ser', como dedica Hegel, y por eso la condición de la existencia humana temporal es formarse, integrarse, convertirse en un ser espiritual capaz de romper con lo inmediato y lo particular, y ascender a la universalidad a través del trabajo y de la reflexión filosófica, partiendo de las propias raíces (1994, p.109).

La formación es quizá el centro del quehacer educativo en la medida que inscribe su sentido en tres aspectos esenciales: (a) La condición de ser persona en el permanente proceso de humanización y constitución de sentido; (b) la formación está orientada a un fin, tiene un propósito, en este sentido la formación busca el desarrollo del hombre como proyecto de sentido compartido; (c) tiene una condición metodológica, es decir, que le pertenece un tipo de racionalidad, desde dónde se piensa y define el acto educativo.

Esto es tan sólo un acercamiento al concepto de formación y sobre el cual señalo que entender la formación es un acontecimiento de sentido individual, en la medida en que es el sujeto en su estar sujeto al mundo quien el últimas termina formándose. Es decir, le da sentido a su ahora, su presente viviente, en diálogo constante entre el pasado, el presente y el futuro. Ello no quiere decir que se aparten las distintas perspectivas sobre la formación. La formación humana, como misión y eje teórico de la pedagogía, trasciende al docente, en tanto hace referencia a su misión de educador y formador de la condición humana de los sujetos con quienes irradia su accionar cotidiano.

Es así que la formación, vista desde el contexto de la escuela, está orientada bajo la idea de realización del ser humano, no como un proceso en la medida de aprendizaje, contenidos, habilidades, tareas, sino más como un conjunto todos éstos, implicados en un todo que permiten, estimulan y promueven los potenciales de la persona.

Es por ello que la formación, entendida desde la lecciones de Husserl, presenta la relación entre sujeto-mundo que permite la constitución de sentido, en la temporalidad de cada uno de los participes de la comunidad escolar. El hecho que el individuo, en singular, sea quien se forme, no quiere decir el derrumbe de cualquier teoría sobre la formación, sino por el contrario, éstas adquieren un campo de compresión más amplio en el que se puede pensar la actividad educativa, como un dar sentido, pero nos asalta la pregunta sobre la manera artificial e instrumental en el que se desarrolla la vida los miembros en la comunidad escolar.

La formación es un concepto que no sólo se resemantiza en la cultura, sino que está inserto en el dinamismo de la vida misma, como un proceso transformador, donde se desarrollan actos formadores. En esta medida, existen no sólo intensiones e intereses formadores e instituciones formadoras, si no que también existen formadores, pues lo que en últimas está en juego son los ideales de humanizar la vida, de dar un sentido más amplio a la inmediatez. Y para ello se promueve la idea de sujeto pensante, crítico y éticamente comprometido, tanto con su destino como con el destino de aquellos con quienes comparte su mundo, en fin, es todo un acontecimiento de constitución de subjetividad, de serpersona.

En el formarse en las actuales dinámicas del mundo, emerge también la pregunta sobre la formación en el vertiginoso y cada vez más apasionante mundo de los desarrollos tecnológicos y comunicacionales: ¿cómo formarse y cómo entender el tiempo escolar con otras perspectivas de tiempo objetivo que rodean y habitan la escuela?

Polisemia No. 13, 94 - 101. Formación, subjetividad y escuela: aproximación fenomenológica al tiempo escolar. Bogotá, ISSN: 1900-4648. Enero - junio de 2012 
La juventud de hoy es el reflejo de un mundo tecno-cultural permeado por los procesos de socialización en distintos niveles de complejidad, en el desplazamiento inevitable de los actores tradicionales como la familia, el maestro, el texto entre otros. Lo que ha llevado, entre otras cosas, a la reorganización de los modelos de crianza, haciendo que los jóvenes sean menos dependientes de los adultos; esto favorecido por la mediación de las tecnologías, donde el movimiento, la velocidad, la sonoridad, las variadas imágenes y el contacto en tiempo real brindan experiencias multisensoriales que las hacen atractivas para la interacción social de los jóvenes. Lo que supone pensar que la temporalidad del espacio escolar no sólo está siendo atravesada por la multiplicidad de temporalidades, sino por otros flujos de tiempo objetivo, tiempo mundo.

Una individualidad que los lleva, en algunos casos, a abstraerse del mundo social "en vivo" y a sumergirse en el mundo virtual, que se antepone a la interacción cara a cara en colectivo y que ha dado cabida a otros aprendizajes no propios de los procesos de socialización primaria que se tejen en la familia y penetran la cultura escolar, incluso con la apropiación de prácticas de otras latitudes, como consecuencia de la importación de costumbres y de saberes que con la internet, la televisión y el celular han favorecido el desplazamiento de las fronteras entre razón e imaginación, entre la formación y la información, entre la realidad y la fantasía, entre la autenticidad y la superficialidad; entre tiempo objetivo y tiempo de la conciencia del tiempo.

Es así que se van constituyendo subjetividades, un nuevo modo de ser joven, que en gran parte depende de los patrones de vida, en contextos donde la familia realiza transacciones sociales, que comparten sus aprendizajes, experiencias, donde el escuchar, el afecto, el reconocimiento están presentes y en la reflexión se contrastan los contenidos de los medios con las vivencias cotidianas, permitiendo asumir una postura crítica ante la multiplicidad de información que favorece la toma de decisiones con conciencia y donde las mediaciones tecnológicas son una herramienta a través de la cual construyen y movilizan el sentido.
Con lo anterior se admite que existen muchas posibilidades de subjetivación, de asumir la vida, en suma, que el proceso no siempre transcurre de igual forma, siendo múltiples las posibilidades de constituirse, de convivir e interactuar de los jóvenes de acuerdo con las condiciones socioculturales y el momento histórico.

El joven como sujeto social está en permanente cambio y es susceptible a ellos, ya que no existe independientemente del mundo que lo rodea; su estructura intelectual, biológica y emocional son el producto de la historia de sus transacciones y la base para sus interacciones sociales presentes y futuras, es decir, sus vivencias del mundo, aunque parezca un campo agonístico (Desser, 1980). En ese devenir su subjetividad se constituye en relación con los aspectos de su propia vida en el dinamismo del mundo.

Una afirmación importante a tener en cuenta es la realizada por Noblia (2004) en su trabajo sobre la ironía en los chats:

La mediación tecnológica tiene efectos inmediatos sobre la constitución de la imagen social y sobre la interacción, especialmente en los casos donde lo único que se expone es la dimensión personal, subjetiva y social de la persona. El riesgo de amenazar la imagen del otro y, en consecuencia poner en riesgo la propia, es alto. Conversar con extraños (escudados todos además en el anonimato del nickname), por escrito y condicionados por las leyes de la inmediatez interlocutiva nos coloca, sin duda, en una situación especial en la que construimos la imagen del otro durante la interacción, pero, 'dado que el único 'territorio' en disputa es la dimensión de lo personal, o sea, aquellos rasgos que los hablantes presentan en sus mensajes y a partir de los cuales se constituyen como sujetos (discursivos), la distinción entre imagen positiva e imagen negativa se torna difusa y poco explicativa' (p. 376).

Los jóvenes requieren que en los diferentes escenarios de interacción se les permita expresar sus sentires, analizar críticamente cualquier evento, tomar postura y decisión; este ejercicio de responsabilidad, de ciudadanía ante cualquier circunstancia de vida, les brinda mayores 
elementos de juicio para tomar decisiones en el escenario virtual, donde se encuentran inmersos en un mundo de información, de estrategias de mercado, de diferentes situaciones cargadas de intereses no siempre positivos.

Martín-Barbero plantea que en el momento actual:

nos encontramos con un ecosistema comunicativo, que es a la sociedad actual tanto o más importante que el sistema verde. El ecosistema comunicativo está compuesto básicamente de dos órdenes de realidades: el orden de las tecnologías de producción, de difusión de los saberes; y las nuevas sensibilidades (2009, p. 26).

Esas sensibilidades les permiten expresarse libre y creativamente a través de múltiples lenguajes en las lógicas propias de las redes y los flujos de información y comunicación, con intenciones que en algunos casos apuntan a la participación social y política, pero no a una política "formal" que parece no interesarles.

Los jóvenes hoy quieren ser vistos, existir para la sociedad, ser reconocidos más que ser representados. Como dice Martín-Barbero:

Hay una relación intrínseca hoy entre el derecho a ser reconocido y el derecho a ser visto, y no con el derecho a ser representado. Los nuevos actores sociales, las mujeres, los jóvenes, los homosexuales, lo que buscan no es quién los represente, lo que buscan es que la sociedad los reconozca en su diferencia subversiva, en la diferencia subversiva que es su propio modo de ser corporal, vital, existencial. Lo que buscan no es que se los represente, es que se los deje ser, es decir, que se los reconozca $(2000$, p. 33).

Los jóvenes buscan ser parte de la sociedad, expresar sus ideas, sus sentires, sus sueños, a su manera, con sonidos, con pinturas, con su cuerpo, expresando desde sus trayectorias de vida.

Los prototipos a seguir han sido en diferentes momentos, músicos, actores y actrices del cine, que constituyen experiencias de subjetivación y de formación en el sentido que orientan un ser siendo en el mundo. En la actualidad son reconocidas figuras como Led Zepelin, Nirvana, Soda Stereo, Menudo, RBD, Daddy Yankee, Dr. Krapula, Kraken, Mago de OZ o Brad Pitt; y películas como La Naranja mecánica, Matrix, El señor de los anillos, La Guerra de las Galaxias, Harry Potter, Crepúsculo; en Colombia, películas como La vendedora de rosas y Rosario Tijeras mostraron una forma particular de reacción de los jóvenes ante las circunstancias sociales propias de comunidades marginales; programas de TV como Friends, y en nuestro país, Francisco el matemático, Sin tetas no hay paraíso, entre otras, convirtiendo a los protagonistas en modelos a seguir por los jóvenes desde su manera de vestir, de bailar, de caminar, de manejar las relaciones afectivas, la sexualidad, las relaciones con el ambiente, la postura ante situaciones de injusticia social, en suma, de relacionarse consigo mismo y con el mundo, de constituirse como sujetos-jóvenes individuales y colectivos.

Pero, ¿qué hacer en estos constantes flujos de tiempos? Con el desarrollo acelerado de las tecnologías de la información se dio paso a un planeta donde las herramientas de la web condensan el tiempo y el espacio, ya los jóvenes no se sitúan únicamente en un espacio geográfico, la dimensión espacio tiempo gira a otras velocidades y en todas las latitudes del planeta. Es decir, que hay mayor intensidad en la vivencia del tiempo objetivo. Al no pertenecer ya a ningún lugar o entorno fijo, la juventud habita crecientemente en esferas culturales y sociales cambiantes y caracterizadas por una pluralidad de lenguajes y culturas. Las comunidades se han reconfigurado a medida que el tiempo y el espacio mutan en redes de ciberespacio múltiples y superpuestas. En los espacios privados y públicos se ve a los jóvenes y a todos los atrapados por las tecnologías aun estando en el mismo lugar que hablan entre ellos a través de buzones de correo electrónico, Messenger y redes sociales como Facebook y Twiter. Finalmente, la formación en la escuela está en una permanente negociación y lecturas del tiempo objetivo en la constitución de la subjetividad de los sujetos de aprendizaje.

Polisemia No. 13, 94 - 101. Formación, subjetividad y escuela: aproximación fenomenológica al tiempo escolar. Bogotá, ISSN: 1900-4648. Enero - junio de 2012 


\section{Referencias bibliográficas}

Agustín (2001). Confesiones. México D.F.: Porrúa.

Garrido-Maturano, A. (2006). El Círculo del tiempo: Observaciones acerca de las relaciones entre sujeto y tiempo en las "Lecciones de la fenomenología de la conciencia interna del tiempo" de E. Husserl. Tópicos, 14, 51-80.

Held, K. (2009). Fenomenología del "tiempo propio" en Husserl y Heidegger. Lámpara de Diógenes. 10 (18 y 19), 9-29.

Husserl, E. (2002). Lecciones de la fenomenología de la conciencia interna del tiempo. Madrid: Trotta.

Mélich, J. (2004). La lección de Auschwitz. Barcelona: Herder.

Desser, D. (1980). Maestro, alumno y tarea. Buenos Aires: Paidós.

Flórez, R. (1994). Hacia una pedagogía del conocimiento. Bogotá: Mc Wright Hill.

Quiceno H. (1996). Rousseau y el concepto de Formación. Educación y Pedagogía, 14 y 15, 66-92.
Martín-Barbero, J. (2000). Cambios culturales, desafios y juventud. Medellín: Corporación Región.

\section{(2002a). Jóvenes: Comunica-} ción e identidad. Recuperado de: http:// ebookbrowse.com/martin-barbero-jovenescomunicacion-e-identidad-pdf-d92918795 .

- (2002b). La educación desde la Comunicación. Recuperado de: http://www. eduteka.org/pdfdir/SaberNarrar.pdf

(2009). Diversidad Cultural y convergencia digital. Recuperado de: http:// www.revistaalambre.com/Articulos/ ArticuloMuestra.asp?Id=36

Noblia, V., Bravo, D \& Briz, A. (2004). La ironía de los chats. Una forma de preservar la imagen y administrar los conflictos en las conversaciones mediadas por computadoras. Recuperado de: http:/ /www. mendeley.com/reserarch/la-ironia-en-laschats-una-forma-de-preservar-la-imagen-yadministrar- los- conflictos- en- las- conversaciones-mediadas-por computadoras/ 